\title{
Efficacy, cost-effectiveness and acceptability of self-help interventions for anxiety disorders: systematic review
}

Catrin Lewis, Jennifer Pearce and Jonathan I. Bisson

\section{Background}

Self-help interventions for psychiatric disorders represent an increasingly popular alternative to therapist-administered psychological therapies, offering the potential of increased access to cost-effective treatment.

\section{Aims \\ To determine the efficacy, cost-effectiveness and acceptability of self-help interventions for anxiety disorders.}

\section{Method}

Randomised controlled trials (RCTs) of self-help interventions for anxiety disorders were identified by searching nine online databases. Studies were grouped according to disorder and meta-analyses were conducted where sufficient data were available. Overall meta-analyses of self-help $v$. waiting list and therapist-administered treatment were also undertaken. Methodological quality was assessed independently by two researchers according to criteria set out by the Cochrane collaboration.

\section{Results}

Thirty-one RCTs met inclusion criteria for the review. Results of the overall meta-analysis comparing self-help with waiting list gave a significant effect size of 0.84 in favour of self-help. comparison of self-help with therapist-administered treatments revealed a significant difference in favour of the latter with an effect size of 0.34 . The addition of guidance and the presentation of multimedia or web-based self-help materials improved treatment outcome.

\section{Conclusions}

Self-help interventions appear to be an effective way of treating individuals diagnosed with social phobia and panic disorder. Further research is required to evaluate the costeffectiveness and acceptability of these interventions.

\section{Declaration of interest}

None.
There is a discrepancy between the burden of mental illness and the availability of cost-effective psychological therapy. This creates a major challenge for healthcare providers. ${ }^{1}$ As a consequence, innovative methods that place less demand on therapist time have been developed. One such method is self-help, an approach that enables self-improvement through the use of health technologies (such as books, CDs, DVDs and websites) with little or no therapist input. This method of treatment delivery shows promise in widening access to psychological therapy and the potential to alleviate the current shortfall in suitably qualified psychological therapists. ${ }^{2}$ According to Bower \& Gilbody, ${ }^{3}$ the use of minimal interventions as part of a stepped-care model relies on assumptions of efficacy, cost-effectiveness and acceptability. Despite recent interest in the use of minimal interventions, ambiguity remains as to whether self-help interventions for anxiety disorders fulfil these criteria. Previous meta-analyses have reported on the efficacy of self-help for conditions including subclinical anxiety problems, ${ }^{4-6}$ but only one has focused on self-help interventions for individuals diagnosed with an anxiety disorder. ${ }^{7}$ However, the review combined studies of bibliotherapy-based self-help interventions and self-help groups, and did not consider self-help delivered by media other than books or leaflets. The current review is the first to bring together the available evidence on the efficacy, cost-effectiveness and acceptability of multimedia self-help interventions for individuals diagnosed with an anxiety disorder.

\section{Method}

\section{Selection criteria}

Randomised controlled trials (RCTs) of self-help interventions aimed at adults formally diagnosed with an anxiety disorder according to DSM or ICD criteria were included. Self-help interventions were defined as those predominantly reliant on the individual bringing about self-change through the use of health technologies including written materials (books, booklets, leaflets), CD-Roms, DVDs, computerised software packages and websites. Interventions of interest comprised pure self-help interventions that involve no therapist input and guided self-help interventions that involve minimal contact with a therapist or trained professional through face-to-face appointments, telephone or email. Studies were only included where a standardised measure of symptomatology related to the target disorder, or anxiety, was used to measure outcome, and where interventions were compared with therapist-administered treatment or a waitinglist control group. Randomised controlled trials of self-help groups, self-help materials adjunctive to therapist-administered psychological therapy, virtual reality exposure unaccompanied by any other self-help material, or one-off presentations of videos or audio tapes aimed only at exposure or relaxation, were not included. Although these interventions offer many of the same advantages as the self-help programmes considered by the review, they are fundamentally distinct in terms of content, delivery and their overall aims and objectives. Consideration of them in the current review would therefore be inappropriate.

\section{Search strategy}

Bibliographic databases were searched from inception using a set of search terms related to anxiety disorders (anxiety, generalised anxiety disorder, panic disorder, panic, phobic disorder, specific phobia, phobia, social phobia, agoraphobia, obsessive-compulsive disorder, obsessive behaviour, post-traumatic stress disorder, acute stress disorder, traumatic stress), combined with a set of terms related to self-help (self-help, self-change, self-directed, self-care, self-management, self-administration, guided self-help, guided 
self-change, minimal contact, minimal therapist contact, reduced contact, self-exposure, internet, web-based, online, computerised cognitive-behavioural therapy (CBT), bibliotherapy, computerised). Databases were searched using both Medical Subject Headings $(\mathrm{MeSH})$ and text terms until the 31 October 2010 incorporating results from MEDLINE, CINAHL, EMBASE, ASSIA, AMED, IBSS, PsycInfo, PILOTS and EBMR. Reference lists of all selected studies were scrutinised for additional RCTs.

\section{Data extraction}

The primary outcome measure for the review was change in anxiety symptoms. The secondary outcome was the rate of drop-out. We also sought to extract information related to the cost-effectiveness of interventions. Acceptability was assessed in terms of any formalised measure of satisfaction.

\section{Data synthesis}

Data were entered into the Cochrane Collaboration's Review Manager 5 software (for Windows) for systematic reviews. Continuous data (change in anxiety symptoms) were analysed using standardised mean differences (SMD) where outcome measures used different scales and weighted mean differences (WMD) where the same scale was used. Relative risk was calculated for categorical outcome measure; 95\% confidence intervals were calculated for all outcomes.

The I statistic was used to assess the degree of true heterogeneity (i.e. heterogeneity which was not the result of chance). Presence or absence of true heterogeneity (i.e. between-studies variability) dictated the appropriate statistical model for the analysis. When results only differed by the sampling error a fixed-effects model was applied. Where studies were truly heterogeneous a random-effects model was used to account for both within- and between-studies variability. The $I$ index is the percentage of the total variability in a set of effect sizes due to true heterogeneity. Here, an $I^{2}$ of less than $30 \%$ was taken to indicate mild heterogeneity and a fixed-effects model was used; $I$ values of $30 \%$ or over dictated use of a random-effects model. ${ }^{8}$

Studies were grouped by target disorder. For inclusion in a specific group more than $70 \%$ of participants were required to meet DSM or ICD criteria for the disorder. All studies included a research assessment to ascertain eligibility. Levels of guidance varied enormously between interventions. Some pure self-help interventions that offered only initial instruction demanded only marginally less clinician time than the least intensive guided self-help interventions. It was felt that a distinction between pure self-help and guided self-help would however be informative. On this basis and in accordance with the definition provided by Newman and colleagues, ${ }^{9}$ studies that included only initial instruction and treatment rationale and/or very brief check-ins to monitor adherence, ensure safety or teach use of the materials, were considered to be pure self-help interventions. We imposed a $1 \mathrm{~h}$ maximum on therapist input for pure self-help interventions. Those including some form of guidance to a lesser degree than traditional therapy for the disorder, ${ }^{9}$ averaging more than $1 \mathrm{~h}$ of therapist time, were classified as guided self-help.

Self-help was compared with waiting-list or therapistadministered CBT. Where two or more self-help conditions were compared with the control, both groups were included to maximise use of data. In this case, the control group sample size was modified in line with guidance issued by the Cochrane Collaboration. ${ }^{10}$

All included studies were quality assessed using the criteria set out by the Cochrane Collaboration, ${ }^{10}$ including: sequence allocation for randomisation, allocation concealment, masking of personnel and assessors, incomplete outcome data, selective outcome reporting and any other notable threats to validity. Two researchers independently assessed each study. Any conflicts were discussed with a third researcher with the aim of reaching a consensus.

\section{Results}

The study selection process is presented in Fig. 1. The search yielded 10304 references. Removal of duplicates gave a total of 8132 for consideration. Abstracts were reviewed and full text copies obtained for 192 potentially relevant studies.

Thirty-one RCTs met the inclusion criteria for this review (online Table DS1). In total 3 studies evaluated an intervention for generalised anxiety disorder (GAD), ${ }^{11-13} 2$ for obsessivecompulsive disorder (OCD), ${ }^{14,15} 14$ for panic disorder, ${ }^{16-29} 1$ for post-traumatic stress disorder (PTSD), ${ }^{30} 8$ for social phobia ${ }^{31-38}$ and 3 for mixed anxiety disorders. ${ }^{39-41}$

\section{Methodological quality of included studies}

A total of 19 of the 31 studies described the process of random allocation in sufficient detail to judge there to have been a low risk of creating bias. $^{12-14,17-19,23,25,28,30-32,34,36-38,40,41}$ Only 17 included adequate reporting of allocation concealment. ${ }^{12,13,17,18,23,25,28,30-32,34,36-38,40,41}$ A total of 17 studies provided sufficient information to deduce that outcome assessors had been adequately masked. ${ }^{11-15,17-19,20,26-31,41}$ Incomplete data were deemed to be appropriately addressed by 24 studies. ${ }^{12-14,17-19,23,25-38,40,41}$ It was unclear whether any of the studies were free of selective reporting. Many of the studies recruited individuals from newspaper advertisements, introducing a possible sampling bias. ${ }^{12,13,17-19,22,26-29,31-34,36-38,41}$ No study reported on side-effects of self-help treatment.

\section{Efficacy}

Generalised anxiety disorder

Three RCTs of self-help interventions for GAD met the inclusion criteria. Bowman et $a l^{11}$ examined the efficacy of a pure self-help

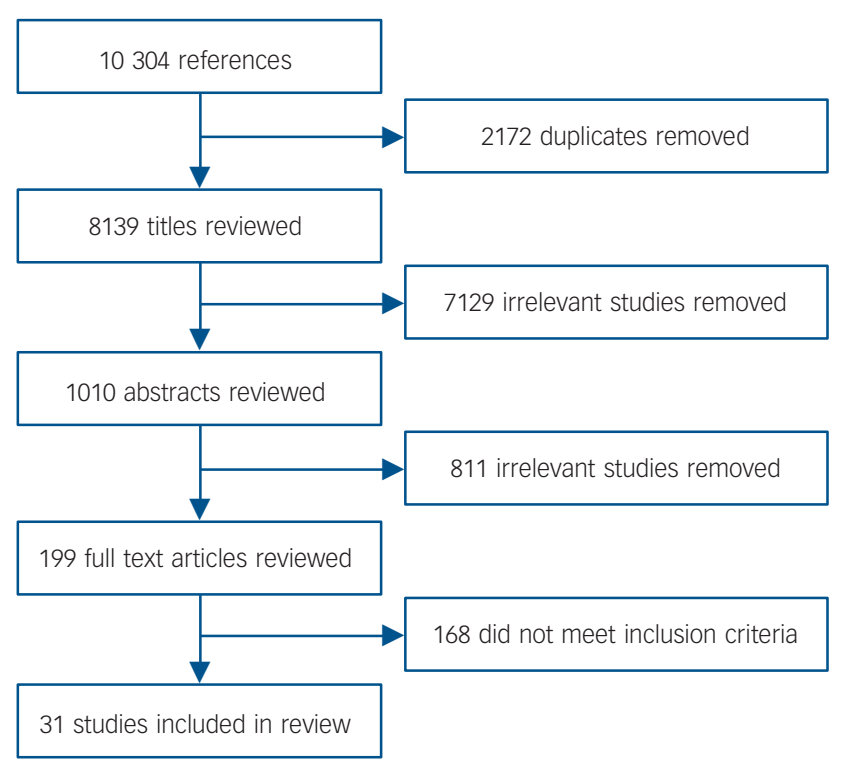

Fig. 1 Search flow chart 
intervention compared with a delayed treatment control group. Titov and colleagues ${ }^{12}$ conducted an RCT of an online guided self-help intervention for GAD called 'Worry' with email guidance. A later RCT of the same programme conducted by Robinson and colleagues ${ }^{13}$ compared the intervention guided by a clinician/technician with a waiting-list control group. A metaanalysis of the three studies revealed a significant difference in favour of the self-help group ( $\mathrm{SMD}=-1.18,95 \% \mathrm{CI}-1.49$ to $-0.86,3$ studies: $n=186$ ). There were no significant differences observed between groups in terms of drop-out rates (odds ratio $(\mathrm{OR})=2.07,95 \%$ CI $0.73-5.89,3$ studies: $n=186$ ). There was however, a trend towards greater drop-out from the self-help condition.

\section{Obsessive-compulsive disorder}

We identified two RCTs of self-help interventions for OCD, both compared self-help adaptations of an exposure and response prevention (ERP) treatment protocol, to the same treatment delivered face to face. ${ }^{14,15}$ Although therapist-administered ERP gave the most favourable results, meta-analysis revealed no significant differences between the two methods of treatment delivery ( $\mathrm{WMD}=1.88,95 \% \mathrm{CI}-0.25$ to $4.01,2$ studies: $n=157)$. There were no significant differences in drop-out rates between the self-help and therapist-administered ERP (OR $=1.21,95 \%$ CI $0.60-2.46,2$ studies: $n=157$ ).

\section{Panic disorder and/or agoraphobia}

Self-help for panic disorder and/or agoraphobia has received significant attention in the literature. Fourteen RCTs were identified. ${ }^{16-29}$ Most of the interventions included some form of guidance ranging from 1.5 to $7 \mathrm{~h}$. Pure self-help interventions were also included, which comprised only brief introductory instruction with no follow-up guidance. Self-help interventions were compared with therapist-administered CBT or waiting-list/ information-only control.

Self-help compared with CBT. Seven RCTs ${ }^{16,18,20,22,23,27,29}$ compared self-help with therapist-administered CBT. Three presented written self-help materials in the form of a book or leaflet. The remainder presented self-help materials on a website. All but one study evaluated guided self-help, with guidance ranging from 3 to $7 \mathrm{~h}$. Meta-analysis of these studies showed no significant difference between self-help and therapist-administered CBT groups ( $\mathrm{SMD}=0.17,95 \% \mathrm{CI}-0.19$ to $0.53,7$ studies: $n=341$ ). There were no significant differences observed between groups when it came to drop-out rates $(\mathrm{OR}=1.10,95 \%$ CI $0.58-2.09$, 7 studies: $n=341$ ).

Self-help $v$. waiting-list /information-only control. Eight studies compared self-help interventions with a waiting-list control group. ${ }^{17,19,21,22,24-26,28}$ Five studies evaluated guided self-help, with guidance ranging from 1.5 to $5.5 \mathrm{~h}$. Self-help materials were presented in a written format or on a website. Three studies took a pure self-help approach, with two providing written materials and one presenting a programme online. One study failed to report adequate data, and was not therefore included in the metaanalysis. ${ }^{24}$ Taken together, these studies suggest that self-help is superior to being placed on a waiting list in terms of symptom reduction. A meta-analysis of the studies showed a significant difference in favour of the self-help condition ( $\mathrm{SMD}=-1.10$, $95 \%$ CI -1.55 to $-0.66,7$ studies: $n=253$ ). There were no significant differences observed between groups when it came to drop-out rates ( $\mathrm{OR}=0.97,95 \% \mathrm{CI} 0.41-2.29,7$ studies: $n=253$ ).

\section{Post-traumatic stress disorder}

To date, there has been only one RCT of a self-help intervention for PTSD. Elhers and colleagues ${ }^{30}$ randomised traumatised individuals to receive cognitive therapy, a self-help booklet presented in a pure self-help format or repeated assessments. This study failed to support the efficacy of pure self-help in the treatment of PTSD. In terms of reduction in traumatic stress symptoms, there was a significant difference in favour of cognitive therapy $(\mathrm{SMD}=2.00,95 \%$ CI $1.33-2.67,1$ study: $n=53)$. There were no significant differences between pure self-help and repeated assessment ( $\mathrm{SMD}=-0.46,95 \% \mathrm{CI}-1.01$ to $0.09,1$ study: $n=52)$. There were no significant differences in drop-out rates in comparison with cognitive therapy $(\mathrm{OR}=7.82,95 \%$ CI $0.39-158.87,1$ study: $n=56)$ or repeated assessment $(\mathrm{OR}=1.62,95 \%$ CI $0.25-10.51$, 1 study: $n=57$ )

\section{Social phobia}

Eight RCTs of self-help for social phobia met our inclusion criteria. ${ }^{31-38}$ All compared bibliotherapy or self-help information presented on a website to a waiting-list control group. A metaanalysis of these studies showed significant differences in terms of the primary outcome measure of social phobia symptomology in favour of the self-help condition $(\mathrm{SMD}=-0.77,95 \% \mathrm{CI}$ -0.94 to $-0.60,8$ studies: $n=591$ ). In terms of drop-out rates, there was a significant difference in favour of the waiting-list condition ( $\mathrm{OR}=2.23,95 \%$ CI 1.18-4.22, 8 studies: $n=591)$.

\section{Mixed anxiety disorders}

Three $\mathrm{RCTs}^{39-41}$ were identified that included primary care participants diagnosed with an assortment of anxiety disorders. These predominantly comprised GAD and panic disorder. Selfhelp took the form of bibliotherapy in two studies, and internet CBT in the other. A meta-analysis of two studies that compared self-help with therapist-administered CBT found a significant difference in favour of the latter (SMD $=0.36$, 95\% CI 0.03 0.69, 2 studies: $n=155)$. There were no significant differences in terms of drop-out rates $(\mathrm{OR}=0.30,95 \%$ CI $0.02-3.69,2$ studies: $n=155)$.

\section{Overall efficacy}

\section{Self-help $v$. waiting list}

Overall, self-help for anxiety disorders did significantly better than waiting list in terms of symptom reduction (SMD $=-0.86,95 \% \mathrm{CI}$ -1.03 to $-0.69,20$ studies: $n=1121$ ). A forest plot of the analysis is presented in Fig. 2. Analyses restricted to studies of guided selfhelp showed greater efficacy ( $\mathrm{SMD}=-0.97,95 \% \mathrm{CI}-1.17$ to $-0.76,12$ studies: $n=666$ ). An analysis considering only web-based and multimedia self-help interventions also showed greater efficacy $(\mathrm{SMD}=-0.90,95 \% \mathrm{CI}-1.13$ to $-0.68,13$ studies: $n=820$ ). In terms of drop-out, there was a significant difference in favour of the waiting-list condition $(\mathrm{OR}=1.98$, 95\% CI 1.26-3.11, 20 studies: $n=1201$ ).

There was a significant difference between self-help and therapist-administered psychological therapy in favour of therapist-administered treatment ( $\mathrm{SMD}=0.34,95 \%$ CI $0.03-$ $0.65,12$ studies: $n=706)$. A forest plot of the analysis is presented in Fig. 3. Removing pure self-help interventions from the analysis resulted in there being no significant difference between guided self-help and therapist-administered treatment, although there remained a trend in favour of the latter $(\mathrm{SMD}=0.31,95 \% \mathrm{CI}$ -0.15 to $0.77,9$ studies: $n=446$ ). An analysis of only web-based and multimedia interventions showed no significant difference 
Experimental

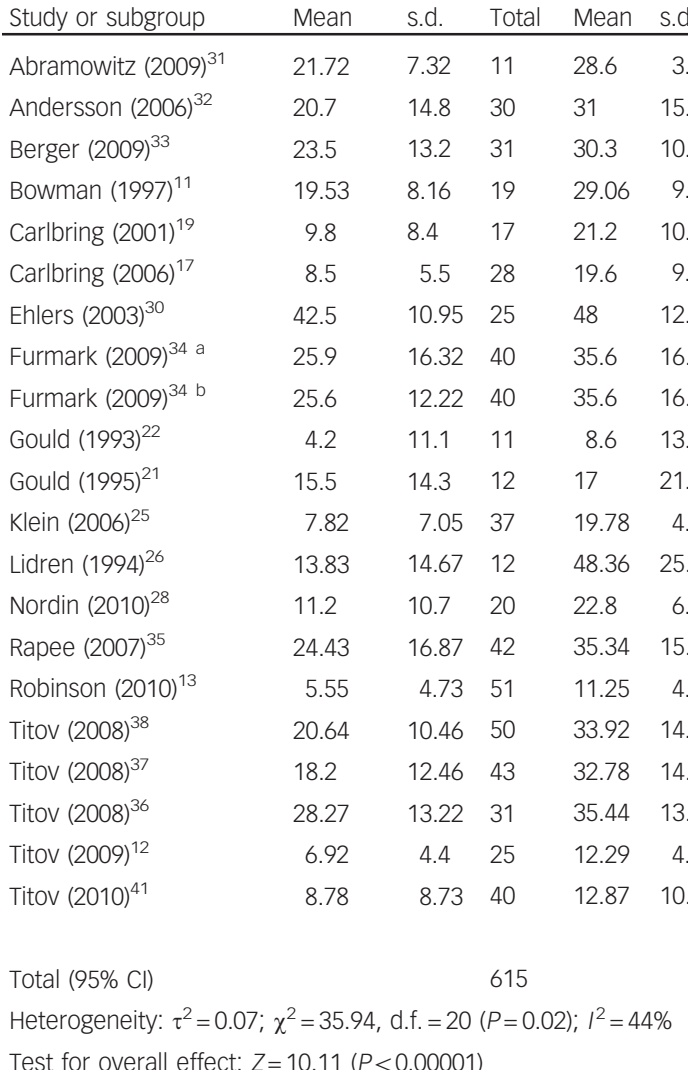

Std. mean difference $\quad$ Std. mean difference

IV, Random, 95\% Cl

$-1.13(-2.07$ to 0.19$)$

$-0.66(-1.17$ to 0.15$)$

$-0.54(-1.11$ to 0.02$)$

$-1.06(-1.75$ to 0.38$)$

$-1.17(-1.89$ to 0.46$)$

$-1.36(-1.94$ to 0.78$)$

$-0.46(-1.01$ to 0.09$)$

$-0.59(-1.14$ to 0.04$)$

$-0.72(-1.28$ to 0.17$)$

$-0.34(-1.19$ to 0.50$)$

-0.08 ( -0.86 to 0.71$)$

$-1.81(-2.55$ to -1.08$)$

$-1.59(-2.53$ to -0.65$)$

$-1.28(-1.97$ to -0.58$)$

$-0.66(-1.10$ to -0.22$)$

$-1.20(-1.63$ to -0.77$)$

$-1.03(-1.46$ to -0.61$)$

$-1.08(-1.53$ to -0.63$)$

$-0.54(-1.03$ to -0.04$)$

$-1.22(-1.84$ to -0.60$)$

$-0.43(-0.88$ to 0.02$)$

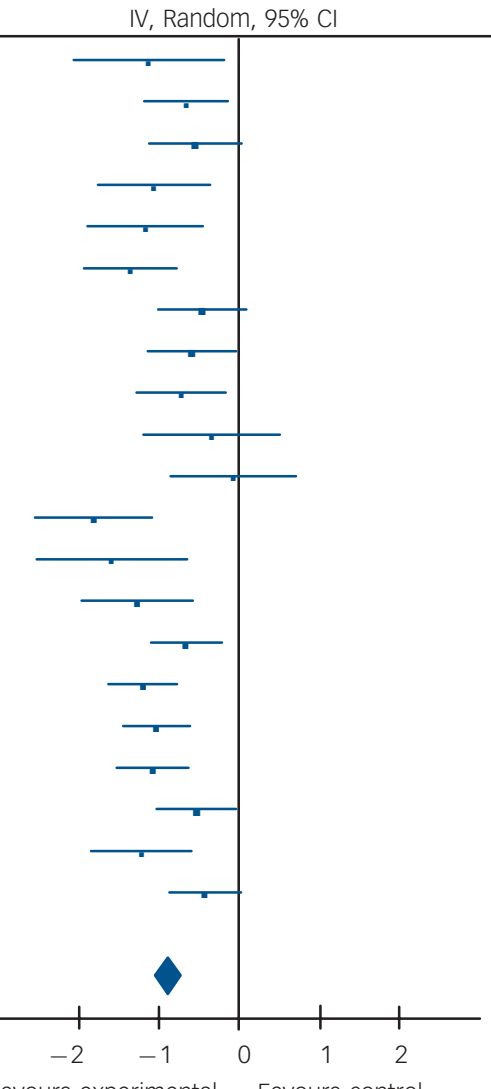

Favours experimental Favours control

Fig. 2 Forest plot of a random effects meta-analysis comparing self-help treatment with waiting list for individuals diagnosed with an anxiety disorder.

a. Written self-help materials presented as a hard copy (e.g. book).

b. Internet-delivered self-help programme.

between self-help and therapist-administered psychological therapy ( $\mathrm{SMD}=0.11,95 \% \mathrm{CI}-0.14$ to $0.36,4$ studies: $n=260$ ). There was no significant difference between the two groups in terms of drop-out (OR=0.79, 95\% CI 0.54-1.18, 12 studies: $n=781)$.

\section{Economic evaluation}

None of the studies included in the review conducted a concurrent economic evaluation of the self-help programme.

\section{Acceptability}

All the RCTs included in this review neglected to assess the acceptability of self-help approaches to those using and delivering the interventions.

\section{Publication bias}

The potential for publication bias was explored using funnel plots. These were visually examined and found to be roughly symmetrical around the mean effect line, giving no indication of publication bias. ${ }^{10}$

\section{Discussion}

\section{Main findings}

Use of self-help interventions as part of a stepped-care treatment model for anxiety disorders depends heavily on their efficacy, cost-effectiveness and acceptability. This review aimed to draw together the available evidence. Results support the ongoing development and implementation of self-help programmes as a potentially effective treatment option for individuals diagnosed with an anxiety disorder. The addition of guidance and the provision of web-based or multimedia materials were also supported.

The results of the overall meta-analysis of self-help interventions compared with waiting list for individuals diagnosed with an anxiety disorder showed a significant difference in favour of self-help, with an effect size of 0.84; a larger effect size of 0.97 was found when considering only guided self-help interventions; and an effect size of 0.90 when considering only web-based and multimedia programmes. This is greater than those found by meta-analyses of subthreshold anxiety problems, which have reported effect sizes of $0.76,{ }^{4} 0.56^{5}$ and $0.62 .^{6}$ It is identical to the effect size reported by den Boer and colleagues in their meta-analysis combining self-help group treatment and bibliotherapy for clinically significant anxiety and depression, which also reported an effect size of $0.84 .^{7}$

Comparison of self-help with therapist-administered treatments revealed a significant difference in favour of the latter, with an effect size of 0.34 . Considering only guided self-help, there was no significant difference between groups with an effect size of 0.31 , however a trend remained in favour of therapistadministered treatment. Similarly, there was no significant difference between groups when only web-based or multimedia interventions were included, with an effect size of -0.11 . 


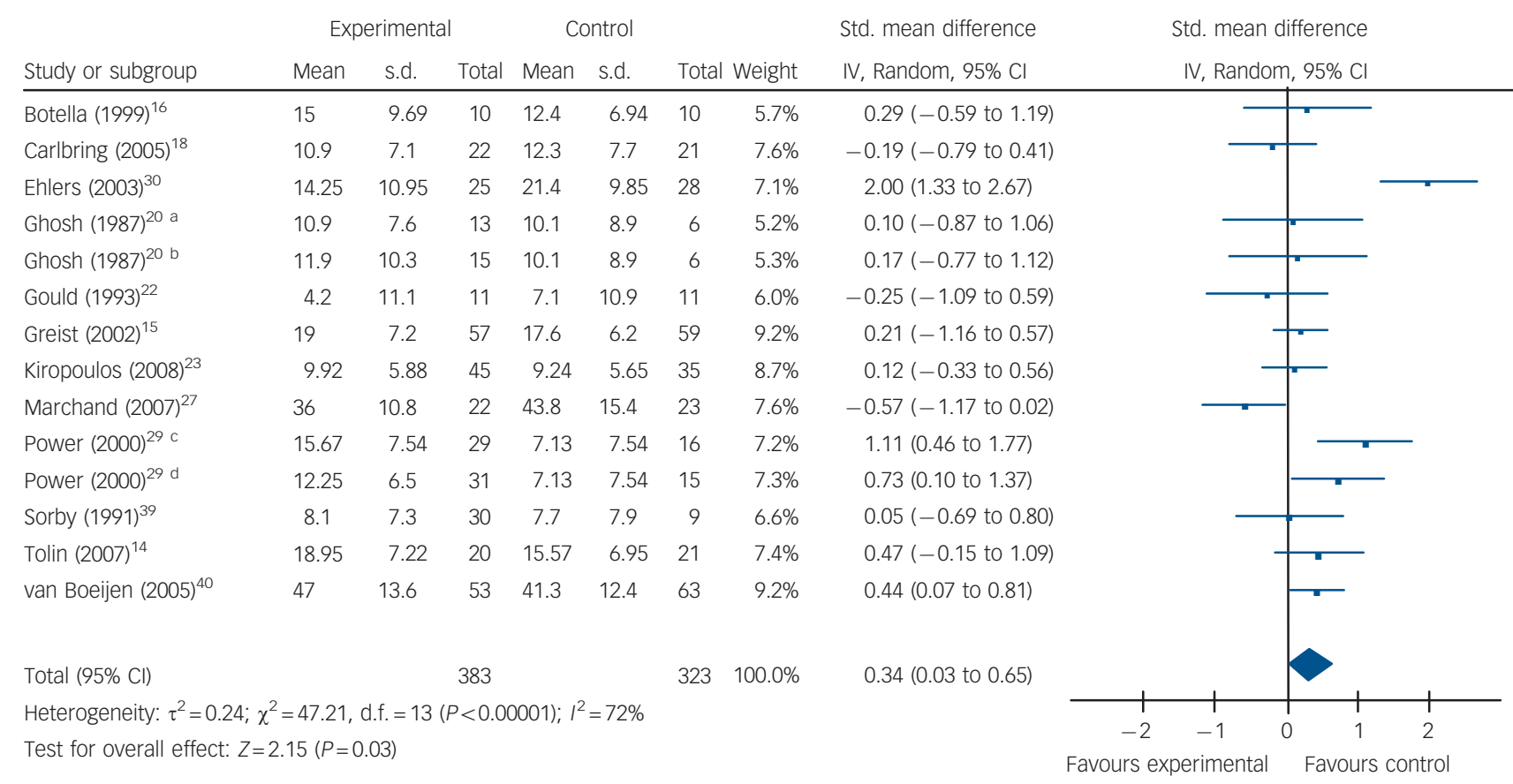

Fig. 3 Forest plot of a random effects meta-analysis comparing self-help treatment with therapist-administered psychological therapy for individuals diagnosed with an anxiety disorder.

a. Written self-help materials presented as a hard copy (e.g. book)

b. Internet-delivered self-help programme.

c. No therapist contact.

d. Up to $2 \mathrm{~h}$ of therapist contact.

In terms of individual disorders, self-help for panic disorder with or without agoraphobia has attracted more attention in the literature than any other anxiety disorder. Meta-analyses revealed significant differences between self-help and waiting-list conditions in favour of the self-help group. Furthermore, no significant differences were observed between self-help treatment and therapist-administered CBT. Although there were fewer available RCTs, meta-analyses indicated results consistent with panic disorder for social phobia and GAD showing a significant difference in favour of self-help in comparison with a waiting list. There were no observed significant differences between self-help and therapist-administered CBT in the case of OCD, although there was a strong trend in favour of therapist-administered CBT. A meta-analysis of two studies of self-help interventions for varied anxiety disorders, however, found a significant difference in favour of the therapist-administered group. There were too few RCTs to conduct a meta-analysis of self-help interventions for PTSD.

Included studies failed to assess the degree to which interventions were acceptable to those using and delivering the programme. Furthermore, none of the studies included economic evaluation. We cannot therefore reach any conclusions regarding the costeffectiveness or acceptability of these interventions. Well-designed RCTs incorporating concurrent economic evaluation and assessment of acceptability are required to address this shortcoming.

\section{Clinical heterogeneity}

There was a great deal of heterogeneity when it came to the selfhelp programmes being evaluated both within and between the anxiety disorders considered. Interventions varied in terms of content, delivery and guidance. Although all were based on cognitive-behavioural techniques, the exact nature of what was included varied from programme to programme. Delivery methods also ranged enormously from simple booklets to advanced multimedia websites. The extent to which use of the self-help material was guided by a trained professional also varied, with some programmes being completely self-explanatory and others providing substantial therapist input.

\section{Limitations}

Only published studies were included in the review, therefore it is important to acknowledge the issue of publication bias. In addition, this review relied only on English-language studies, which limits generalisability. There is considerable work ongoing in both The Netherlands and Scandinavia, some of which may not have been considered by this review and consequently affected the results.

Sample sizes were small. It can therefore be argued that the absence of significant differences between self-help and therapist-administered treatment in some of the meta-analyses may represent a lack of statistical power rather than true equivalence of the approaches. This is particularly pertinent in the meta-analysis for OCD. Larger-scale studies evaluating the efficacy of self-help interventions are required, especially as all but two of the anxiety disorder categories were represented by only one to three studies.

The quality of individual studies is of importance when interpreting the results of any meta-analysis. There are several criticisms that can be made of the RCTs included in this review. First, participants were often recruited by advertisements in newspapers or magazines. ${ }^{11,18,19,22,27,36-38}$ This may limit generalisability. However, it can be argued that recruitment via the media results in a more representative sample than one limited to the primary care population. Indeed, the current emphasis on Increasing Access to Psychological Therapies (IAPT) has affected a change in the characteristics of the treated population by advocating self-referral. On this basis it is intuitive to recruit from 
a wider population base when evaluating self-help interventions. This may be a worthwhile avenue for future research to explore. Second, many of the included studies demonstrated a lack of independent evaluation. Several of the programmes were evaluated by the programme developers themselves ${ }^{16,21,22,32,35}$ as opposed to independent researchers.

Little is known about factors that determine the effectiveness of a self-help programme. Factors associated with programme content, delivery or study populations have not been adequately explored. It is difficult to ascertain what effect, if any, these variables had on the outcomes. Further research is required to determine the active ingredients of successful interventions. Newman ${ }^{42}$ highlighted the observation that self-help interventions are not appropriate for everyone, suggesting that greater emphasis should be placed on the determination and utilisation of individual predictors of treatment response. Information is lacking with respect to the characteristics of individuals who may benefit.

Many of the included studies failed to evaluate the extent to which individuals used self-help materials. This precluded assessment of the effects of increased adherence. Outcome has previously been associated with greater use of self-help materials, ${ }^{32}$ with studies reporting strong dose-response relationships. ${ }^{43}$ Adherence may be improved with increased levels of guidance, which links to a documented superiority of guided self-help in comparison with pure self-help. ${ }^{36}$ There is, however, no agreement as to an optimal level. Randomised controlled trials of guided self-help interventions including concurrent economic evaluation would assist in determining the level of guidance at which cost-effectiveness is optimal.

Studies that evaluated the relative efficacy of pure self-help compared with guided self-help found that the addition of guidance gave superior results. Pure self-help interventions did, however, perform well on the whole, and given their lower cost and greater accessibility, may have a place in a stepped model of care. We cannot assume, however, that outcome would be equivalent if an individual were to purchase a self-help book or access materials online. Inclusion in a study potentially motivates use of materials through creation of deadlines and the knowledge that adherence and/or symptom severity will be assessed. ${ }^{44}$

\section{Implications}

There is evidence that self-help interventions are effective in the treatment of social phobia and panic disorder. This provides support for the inclusion of self-help interventions in stepped care treatment models for these disorders. Methods of providing home treatment for these individuals, who are likely to be fearful of going out in public, has potential to increase engagement.

Further high-quality RCTs that include economic evaluation are now required, particularly of programmes addressing PTSD, OCD, GAD and specific phobias, which have received less attention in the literature than social phobia and panic disorder. Further research is also required to formally evaluate the acceptability of self-help approaches from the perspective of both patient and professional. This might take the form of qualitative research to explore attitudes and opinions. Evaluation of self-help interventions in the context of a stepped-care model would also be valuable.

The self-help approach has received growing recognition in a climate of limited resources and pressure on psychological services. This review confirms its potential for effectively treating individuals diagnosed with an anxiety disorder and supports the ongoing development of self-help programmes with potential for inclusion in stepped care treatment models.
Catrin Lewis, BSC, Jennifer Pearce, BSC, Jonathan I. Bisson, DM, Department of Psychological Medicine, Cardiff University, Cardiff, UK

Correspondence: Catrin Lewis, Department of Psychological Medicine, Cardiff University, Monmouth House, University Hospital of Wales, Heath Park, Cardiff, CF14 4XW, UK. Email: LewisCE7@cf.ac.uk

First received 15 Sep 2010, final revision 29 Mar 2011, accepted 26 May 2011

\section{References}

1 Goldberg D, Gournay K. The General Practitioner, the Psychiatrist and the Burden of Mental Health Care. Institute of Psychiatry, 1997.

2 Andersson G, Bergström J, Holländare F, Carlbring PER, Kaldo V, Ekselius L. Internet-based self-help for depression: randomised controlled trial. Br J Psychiatry 2005; 187: 456-61.

3 Bower P, Gilbody S. Stepped care in psychological therapies: access, effectiveness and efficiency. Narrative literature review. Br J Psychiatry 2005; 186: $11-7$.

4 Gould RA, Clum GA. A meta analysis of self help treatment approaches. Clin Psychol Rev 1993; 13: 169-86.

5 Marrs RW. A meta analysis of bibliotherapy studies. Am J Community Psychol 1995; 23: 843-70.

6 Hirai M, Clum GA. A meta-analytic study of self-help interventions for anxiety problems. Behav Ther 2006; 37: 99-111.

7 den Boer PCAM, Weirsma D, van Den Bosch RJ. Why is self-help neglected in the treatment of emotional disorders? Psychol Med 2004; 34: 959-71.

8 Centre for Reviews and Dissemination. Systematic Reviews: CRD's Guidance for Undertaking Reviews in Health Care. University of York, 2009.

9 Newman MG, Erickson T, Przeworski A, Dzus E. Self-help and minimalcontact therapies for anxiety disorders: Is human contact necessary for therapeutic efficacy? J Clin Psychol 2003; 59: 251-74.

10 Higgins JPT, Green S. Cochrane Handbook For Systematic Reviews of Interventions (Version 5.0.2). The Cochrane Collaboration, 2009.

11 Bowman D, Scogin F, Floyd M, Patton E, Gist L. Efficacy of self-examination therapy in the treatment of generalized anxiety disorder. J Counsel Psychol 1997; 44: 267-73.

12 Titov N, Andrews G, Robinson E, Schwencke G, Johnston L, Solley K, et al. Clinician-assisted Internet-based treatment is effective for generalized anxiety disorder: randomized controlled trial. Aust N Z J Psychiatry 2009; 43: 905-12.

13 Robinson E, Titov N, Andrews G, McIntyre K, Schwencke G, Solley K. Internet treatment for generalized anxiety disorder: A randomized controlled trial comparing clinician vs. technician assistance. PLOS One 2010; 5: e10942.

14 Tolin DF, Hannan S, Maltby N, Diefenbach GJ, Worhunsky P, Brady RE, et al. A randomized controlled trial of self-directed versus therapist-directed cognitive-behavioral therapy for obsessive-compulsive disorder patients with prior medication trials. Behav Ther 2007; 38: 179-91.

15 Greist JH, Marks IM, Baer L, Kobak KA, Wenzel KW, Hirsch MJ, et al. Behavior therapy for obsessive-compulsive disorder guided by a computer or by a clinician compared with relaxation as a control. J Clin Psychiatry 2002; 63: 138-45.

16 Botella C, Garcia-Palacios A. The possibility of reducing therapist contact and total length of therapy in the treatment of panic disorder. Behav Cogn Psychother 1999; 27: 231-47.

17 Carlbring $\mathrm{P}$, Bohman S, Brunt S, Buhrman M, Westling BE, Ekselius L, et al Remote treatment of panic disorder: a randomized trial of internet-based cognitive behavior therapy supplemented with telephone calls. Am J Psychiatry 2006; 163: 2119-25.

18 Carlbring $\mathrm{P}$, Nilsson-Ihrfelt E, Waara J, Kollenstam C, Buhrman M, Kaldo V, et al. Treatment of panic disorder: live therapy vs. self-help via the Internet. Behav Res Ther 2005; 43: 1321-33.

19 Carlbring $P$, Westling B, Ljungstrand P, Ekselius L, Andersson G. Treatment of panic disorder via the internet: a randomized trial of a self-help program. Behav Ther 2001; 32: 751-64.

20 Ghosh A, Marks IM. Self-treatment of agoraphobia by exposure. Behav Ther 1987; 18: 3-16.

21 Gould RA, Clum GA. Self-help plus minimal therapist contact in the treatment of panic disorder: a replication and extension. Behav Ther 1995; 26: 533-46.

22 Gould RA, Clum GA, Shapiro D. The use of bibliotherapy in the treatment of panic: a preliminary investigation. Behav Ther 1993; 24: 241-52. 
23 Kiropoulos LA, Klein B, Austin DW, Gilson K, Pier C, Mitchell J, et al. Is internet-based $\mathrm{CBT}$ for panic disorder and agoraphobia as effective as face-to-face CBT? J Anxiety Disord 2008; 22: 1273-84.

24 Klein B, Richards JC. A brief Internet-based treatment for panic disorder. Behav Cogn Psychother 2001; 29: 113-7.

25 Klein B, Richards JC, Austin DW, Klein B, Richards JC, Austin DW. Efficacy of internet therapy for panic disorder. J Behav Ther Exp Psychiatry 2006; 37: $213-38$

26 Lidren DM, Watkins PL, Gould RA, Clum GA, Asterino M, Tulloch HL. A comparison of bibliotherapy and group therapy in the treatment of panic disorder. J Consult Clin Psychol 1994; 62: 865-9.

27 Marchand A, Todorov C, Borgeat F, Pelland ME. Effectiveness of a brief cognitive behavioural therapy for panic disorder with agoraphobia and the impact of partner involvement. Behav Cogn Psychother 2007; 35: 613-29.

28 Nordin S, Carlbring P, Cuijpers P, Andersson G. Expanding the limits of bibliotherapy for panic disorder: randomized trial of self-help without support but with a clear deadline. Behav Ther 2010; 41: 267-76.

29 Power KG, Sharp DM, Swanson V, Simpson RJ. Therapist contact in cognitive behaviour therapy for panic disorder and agoraphobia in primary care. Clin Psychol Psychother 2000; 7: 37-46.

30 Ehlers A, Clark DM, Hackmann A, McManus F, Fennell M, Herbert C, et al. A randomized controlled trial of cognitive therapy, a self-help booklet, and repeated assessments as early interventions for posttraumatic stress disorder. Arch Gen Psychiatry 2003; 60: 1024-32.

31 Abramowitz JS, Moore EL, Braddock AE, Harrington DL. Self-help cognitivebehavioral therapy with minimal therapist contact for social phobia: a controlled trial. J Behav Ther Exp Psychiatry 2009; 40: 98-105.

32 Andersson G, Carlbring P, Holmstrom A, Sparthan E, Furmark T, Nilsson-Ihrfelt $E$, et al. Internet-based self-help with therapist feedback and in vivo group exposure for social phobia: a randomized controlled trial. J Consult Clin Psychol 2006; 74: 677-86.

33 Berger T, Hohl E, Caspar F. Internet-based treatment for social phobia: a randomized controlled trial. J Clin Psychol 2009; 65: 1021-35.
34 Furmark T, Carlbring P, Hedman E, Sonnenstein A, Clevberger P, Bohman B, et al. Guided and unguided self-help for social anxiety disorder: randomised controlled trial. Br J Psychiatry 2009; 195: 440-7.

35 Rapee RM, Abbott MJ, Baillie AJ, Gaston JE. Treatment of social phobia through pure self-help and therapist-augmented self-help. Br J Psychiatry 2007; 191: 246-52.

36 Titov N, Andrews G, Choi I, Schwencke G, Mahoney A. Shyness 3: randomized controlled trial of guided versus unguided Internet-based CBT for social phobia. Aust N Z J Psychiatry 2008; 42: 1030-40.

37 Titov N, Andrews G, Schwencke G. Shyness 2: treating social phobia online: replication and extension. Aust N Z J Psychiatry 2008; 42: 595-605.

38 Titov N, Andrews G, Schwencke G, Drobny J, Einstein D. Shyness 1: distance treatment of social phobia over the Internet. Aust N Z J Psychiatry 2008; 42: 585-94.

39 Sorby NG, Reavley W, Huber JW. Self help programme for anxiety in general practice: controlled trial of an anxiety management booklet. Br J Gen Pract 1991; 41: 417-20.

40 van Boeijen CA, van Oppen $P$, Van Balkom AJLM, Visser S, Kempe $P$, Blankenstein $\mathrm{N}$, et al. Treatment of anxiety disorders in primary care practice: a randomised controlled trial. Br J Gen Pract 2005; 55: 763-9.

41 Titov N, Andrews G, Johnston L, Robinson E, Spence J. Transdiagnostic internet treatment for anxiety disorders: a randomized controlled trial. Behav Res Ther 2010; 48: 890-9.

42 Newman MG. Recommendations for a cost-offset model of psychotherapy allocation using generalized anxiety disorder as an example. J Consult Clin Psychol 2000; 68: 549-55.

43 Bower P, Richards D, Lovell K. The clinical and cost-effectiveness of self-help treatments for anxiety and depressive disorders in primary care: a systematic review. Br J Gen Pract 2001; 51: 838-45.

44 Loeb KL, Wilson GT, Gilbert JS, Labouvie E. Guided and unguided self-help for binge eating. Behav Res Ther 2000; 38: 259-72. 